\title{
The Time Reversal Invariance Experiment at COSY (TRIC)
}

\section{Eversheim ${ }^{1}$}

Helmholtz-Institut für Strahlen- und Kernphysik, University Bonn

Nussallee14-16, Bonn, D-53115, German

E-mail: eversheidhiskp.uni-bonn.de

\section{Yu. Valdau}

Institut für Kernphysik, Forschungszentrum Jülich

Jülich, D- 52425, Germany

E-mail: y.valdaulfz-juelich.de

\section{B. Lorentz}

Institut für Kernphysik, Forschungszentrum Jülich

Jülich, D- 52425, Germany

E-mail: b. Iorentzafz-juelich.de

\begin{abstract}
One problem of the Big Bang model is the dominance of matter over antimatter in the Universe. The Standard Model prediction for the ratio of baryons over antibaryons deviates from the astrophysical observations by eight orders of magnitude. To explain this discrepancy a strong CP or T-violation must be found. A possible discovery of a T-symmetry violation in the system of baryons would be a strong indication for the existence of physics beyond the Standard Model.

At the Cooler-Synchrotron COSY-Jülich, using a polarized proton beam and a tensor polarized deuterium target, we can measure an unique true (T-odd, P-even) null observable $\mathrm{A}_{\mathrm{y}, \mathrm{xz}}$. This will serve as a new test of the T-symmetry violation in a baryonic system. In order to measure the forward scattering amplitude, the TRIC experiment is conceived as an internal transmission experiment in the storage ring with a storage-cell, fed by a tensor polarized deuteron beam of the PAX atomic beam source. By flipping the spin of beam or target a possible T-symmetry violation reveals itself by different total cross-sections and therefore by a different decrease of the intensity of the circulating beam in the ring. Thus, for the TRIC experiment COSY will serve as an accelerator, storage ring, detector, and ideal zero degree spectrometer.
\end{abstract}

The 26th International Nuclear Physics Conference

11-16 September, 2016

Adelaide, Australia

\footnotetext{
${ }^{1}$ Speaker 


\section{Introduction}

Searches for processes with a symmetry violation are an important tool to track down physics beyond the Standard Model (SM), since the standard model ${ }^{1}$ of fundamental particles is based on a concept of symmetry. Searches for these processes seem necessary in order to explain the baryon asymmetry i.e. matter-antimatter asymmetry of the universe ${ }^{2}$. The baryon asymmetry parameter - the ratio of the baryon-antibaryon difference over the number of cosmic background radiation photons - can be derived to be $6 \cdot 10^{-10}$ from the WMAP and COBE missions $^{3}$. Presently only CP violating processes are associated with matter antimatter asymmetry. These effects are incorporated into the SM using a phase in the Cabibbo-Kobayashi-Maskawa matrix for three generations of quarks or a theta term in the Lagrangian of quantum chromodynamics. The baryon asymmetry parameter is expected within the SM to be of the order of $10^{-18}$. Thus, physics beyond the SM seems to be necessary to explain the baryon asymmetry of the universe. Up to now no processes are discovered which violate the CPT symmetry. Hence, assuming CPT symmetry, $\mathrm{CP}$ violation implies a $\mathrm{T}$ violation. The $\mathrm{T}$ symmetry violation has been discovered in $\mathrm{K}$ and $\mathrm{B}$ meson systems, but has never been observed in a system of hadrons.

In general a time invariance test addresses physics of the very early universe long before direct observations became possible, once the charged particles in the hot plasma from the Big Bang could recombine after about 370000 years. Only then, photons could escape the plasma, since Thomson scattering from charged particles ceased.

An Electric Dipole Moment (EDM) of an elementary particle simultaneously violates both $\mathrm{P}$ and $\mathrm{T}$ invariance. It serves as a very sensitive probe for the physics beyond the SM. It is possible to link an EDM of an elementary particle to a T invariance and hence CP violation, if CPT symmetry is assumed. At present the most sensitive test in this respect is the upper limit of an electric dipole moment of the neutron ${ }^{4}\left(1.6 \cdot 10^{-26} \mathrm{ecm}\right)$, which implies a strength of PT-violation of $g_{\mathrm{PT}} \sim 10^{-11}$. This value seems already too small to explain the baryon asymmetry of the universe. Thus, a different channel, for which $\mathrm{P}$ holds and only $\mathrm{T}$ is violated seems to be worth while to be explored. In this case an upper limit for $\mathrm{g}_{\mathrm{T}}<10^{-4}$ can be derived ${ }^{5}$ from the $\mathrm{n}$-EDM. It is worth noting that EDM and TRIC probe different extensions of the SM. This can be seen if the Lagrangian $\mathrm{L}_{\mathrm{SM}}$ of the $\mathrm{SM}$ is extended by a T-odd, P-odd (EDM) part $\mathrm{L}_{\mathrm{TVPV}}$ and a T-odd, $\mathrm{P}$ even part $\mathrm{L}_{\mathrm{TVPC}}$ (TRIC):

$$
\mathrm{L}=\mathrm{L}_{\mathrm{SM}}+\alpha_{\mathrm{TVPV}} \mathrm{L}_{\mathrm{TVPV}}+\alpha_{\mathrm{TVPC}} \mathrm{L}_{\mathrm{TVPC}} \quad \text { with } \quad \alpha_{\mathrm{TVPV}} \text { and } \alpha_{\mathrm{TVPC}} \ll<1
$$

The TRIC experiment ${ }^{6}$ will test in a double polarized proton-deuteron scattering a T-odd, Peven interaction. Fig. 1 shows the situation in the c.m.-system, with: a) The basic system is shown. b) The time reversal operation is applied (spins and momenta are reversed and the particles are exchanged). In order to have a direct comparison between situation a) and b), two rotations $\mathrm{R}_{\mathrm{x}}$ or $\mathrm{R}_{\mathrm{y}}$ by $180^{\circ}$ about the $\mathrm{x}$ - or $\mathrm{y}$-axes are applied, leading to the situations c) and $\mathrm{d}$ ), respectively. This is allowed, since the scattering process is invariant under spatial rotations.

Conzett ${ }^{7}$ showed that the total cross-section of scattering vector polarized protons off tensor polarized deuterium atoms is a true P-even T-odd null observable, i.e. the total correlation $\mathrm{A}_{\mathrm{y}, \mathrm{xz}}$. A null experiment is favourable, since systematic errors can be identified by switching off an essential beam and/or target parameter i.e. the polarization. Consequentely, the result of the measurement has then to be null, otherwise residual systematic errors are revealed and can be identified.. In order to keep general errors as small as possible, the experiment should be 
performed at an energy for which its sensitivity is highest. In this respect two independent theoretical calculations suggest to perform TRIC below $200 \mathrm{MeV}$. M. Beyer ${ }^{8}$ estimated this energy to be about $135 \mathrm{MeV}$ from the mass of the $\rho$-meson involved in the P-even T-odd process, whereas calculations by Yu. Uzikov ${ }^{9}$ were performed in the framework of Glauber theory.

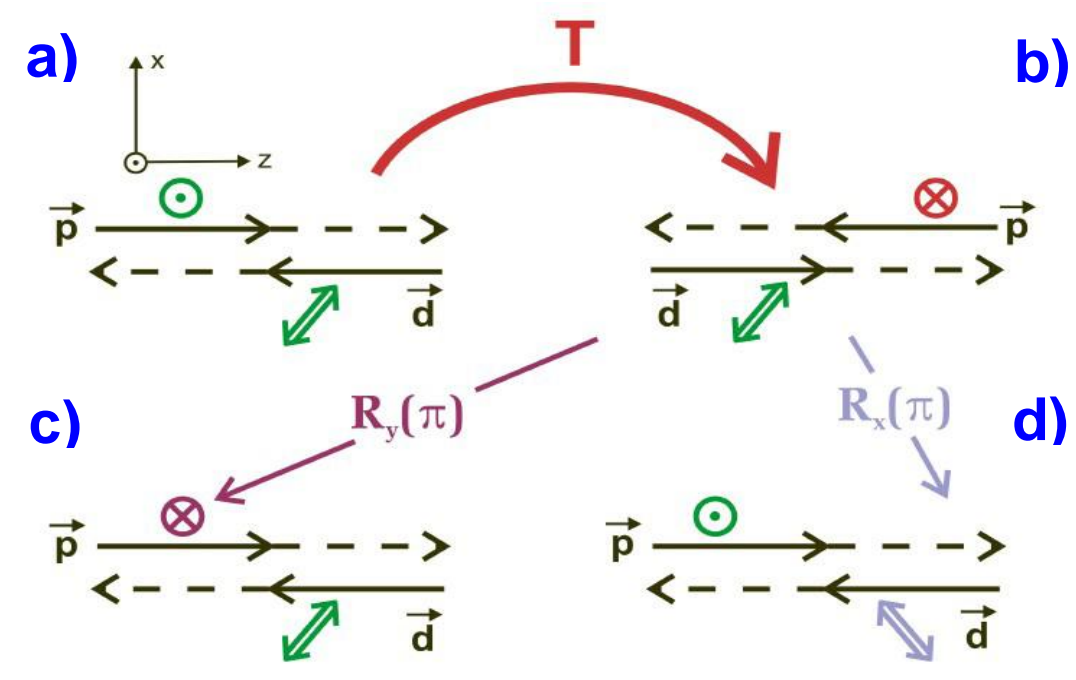

Fig. 1 Scheme of a time-reversed situation that is prepared by either a proton or a deuteron spin-flip. $\odot$ Proton spin up (y-direction), $\otimes$ Proton spin down, $\Leftrightarrow$ Deuteron tensor polarization

Usually total cross-sections are measured in an external experiment with a front detector, the high density target, and the back detector. The front detector measures the incoming particles and the back detector measures all scattered particles in a solid angle corresponding to $4 \pi$ in the center of mass system. This has to be true for all kinds of particles at any possible energy. Therefore, the accuracy of these experiments is limited by the quality of calibration. On the other hand the optical theorem ${ }^{10}$ can be utilized rendering the problem to a precise measurement of the forward scattering amplitude. In this case only particles under $0^{\circ}$ in a solid angle which tends to zero should be considered. Hence, the measurement will starve from statistics. Alternatively the solid angle can be opened, but then the measurement will not be model independent any more. Still the problematic nature of the calibration remains.

In the TRIC experiment a vector polarized proton beam will interact with an internal tensor polarized deuterium target placed in one of the straight sections of the COSY accelerator. The target density will be enhanced my means of a target-cell supplied by the PAX collaboration. The beam passes some $10^{5}$ times per second through the target. Somewhere in the ring the current of the circulating protons is measured by a Beam Current Transformer ${ }^{11}$ (BCT) or a Fast Current Transformer (FCT). Each of these devices serves as front detector before the beam passed through the target and serves as back detector once the beam passes through these devices the next time. At the same time the back detector becomes the front detector for the next turn of the circulating protons. With this trick, the calibration problem is essentially avoided, 
since the total cross-section is calculated from the slope of the decreasing circulating proton current rather than from any absolute current value.

Since COSY can only accept forward scattered particles in an extremely small solid angle, it serves for this experiment not only as an accelerator but also as an ideal zero degree spectrometer allowing for the application of the optical theorem. These total cross-section measurements are inherently free from uncertainties due to final state interactions arising in many other experiments with nuclear beams that test fundamental symmetries ${ }^{12}$. The total cross-section of the double polarized proton-deuteron interaction will - depending on the spin setting of protons and deuterons - result in slopes of different steepness of the circulating COSY beam current. Using this novel method to measure total cross-sections and the simplest spin- $1 / 2-$ spin- 1 system it is possible to improve an upper limit on the strength of the P-even T-odd interaction by an order of magnitude compared to the value previously discussed in literature ${ }^{13}$.

\section{Some Experimental Details}

The general set-up is depicted in Fig. 2

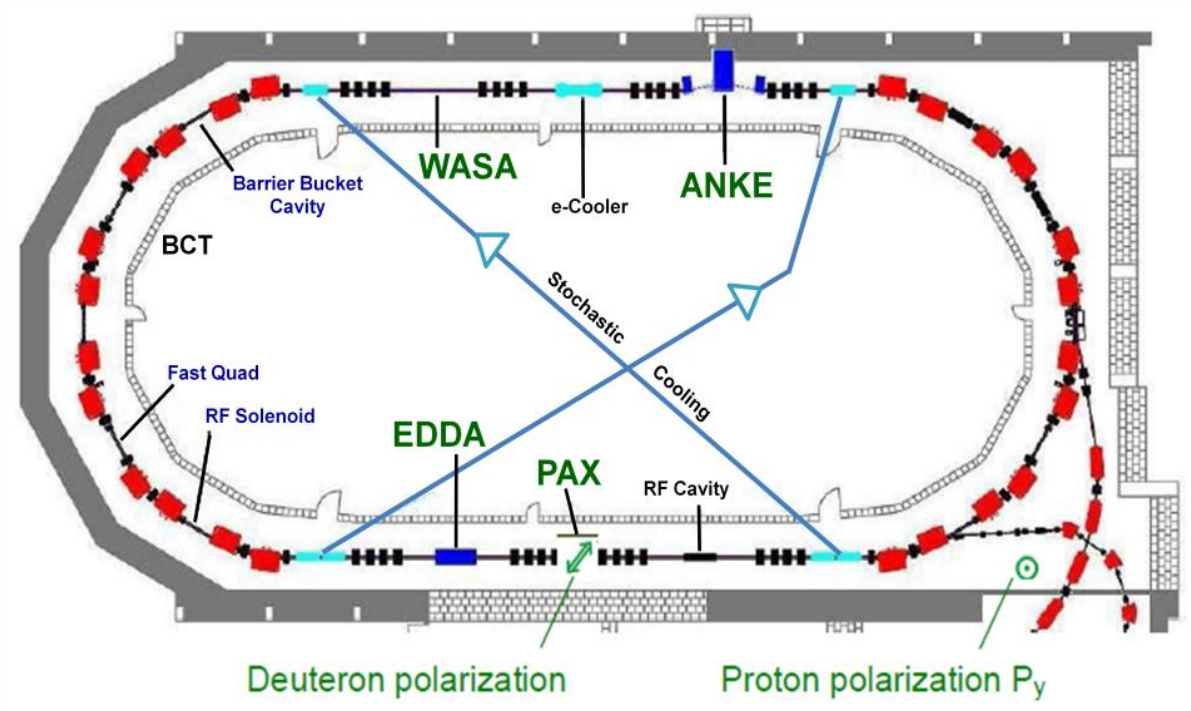

Fig. 2 The TRIC experiment will take place at the PAX target location.

In contrast to external total cross-section measurements the TRIC experiment constitutes an internal measurement. Since the losses due to scattering in the target-cell are measured by the decrease of the beam intensity as the beam circulates through the target, the current measurement has to be utmost stable and of high resolution. Given the time interval $\Delta \mathrm{t}$ between two consecutive current measurements on a slope [s], the spin flip period $\mathrm{h}$ of the target [h], the total measuring time $\mathrm{H}[\mathrm{h}]$, and $\delta \mathrm{I}$ the error in the current measurement in the interval $\Delta \mathrm{t}[\mathrm{A}]$, then the minimal error of the observable in question can be expected to be :

$$
\delta \mathrm{A}_{\mathrm{y}, \mathrm{xz}}^{\text {meas }} \propto \frac{\sqrt{\Delta \mathrm{t}}}{\mathrm{h} \sqrt{\mathrm{H}}} \delta \mathrm{I}
$$

stressing the importance of a small $\delta \mathrm{I}$ for the error of the total spin correlation $\mathrm{A}_{\mathrm{y}, \mathrm{xz}}$. Moreover, a small $\delta$ I reduces the spin-flip period too, which in turn reduces systematic errors due to drifts. The minimal spin flip period $\mathrm{h}_{\min }$ can be derived from the condition, that the measured error $\delta \mathrm{A}_{\mathrm{y}, \mathrm{xz}}$ equals the calculated error from the shot-noise of the scattered particles off the target due to the known total cross-section in p-d scattering. It can be shown that: 


$$
\mathrm{h}_{\min } \propto \delta \mathrm{I}
$$

Recently the COSY staff succeeded in providing polarized bunched beams with sufficiently long life- and spin coherence times (some hours). This allows for a simpler and therefore more accurate measurement device (FCT) compared to the presently installed device (BCT) for measuring coasting (continuous DC) beams in COSY. In this respect we expect a reduction of systematic error contributions by more than an order of magnitude.

\section{Error Considerations}

In Table 1 all observables in $\overrightarrow{\mathrm{p}}-\overrightarrow{\mathrm{d}}$ scattering are listed.

Table 1. Polarization observables of the total cross-section in $\vec{p}-\vec{d}$ scattering. The first index refers to the proton polarization, the second and third index refers to the deuteron vector- and tensor-polarization. All quantities with a hat cancel, since they are odd with respect to a rotation around the $\mathrm{z}$-axis $\left(\mathrm{R}_{\mathrm{z}}\right.$-odd). All quantities which are doubly underlined are $\mathrm{P}_{\mathrm{z}}$-odd

\begin{tabular}{|c|c|c|c|c|c|c|c|}
\hline Line & & & servab & & & & Line cancels because of: \\
\hline 1 & $\mathrm{I}_{\mathrm{o}, \mathrm{o}}$ & $\underline{\underline{\hat{A}}}_{\underline{0 . x}}$ & $\mathrm{~A}_{\mathrm{o}, \mathrm{y}}$ & $\underline{\underline{\hat{A}_{0, z}}}$ & & & protonspinflip \\
\hline 2 & $\underline{\underline{\hat{\hat{A}}}} \underline{\underline{x}, 0}$ & $\mathrm{~A}_{\mathrm{x}, \mathrm{x}}$ & $\underline{\underline{\mathrm{A}_{x}} \underline{\underline{y}}}$ & $\underline{\underline{\hat{\hat{A}}}}_{\mathrm{x}, \mathrm{Z}}$ & & & $P_{x}$ negligible for protons \\
\hline 3 & $\underline{\underline{\hat{A}}}_{y, 0}$ & $\underline{\underline{\mathrm{A}_{\mathrm{y}, \underline{x}}}}$ & $\mathrm{~A}_{\mathrm{y}, \mathrm{y}}$ & $\underline{\underline{\hat{A}}}_{\underline{\underline{y}, z}}$ & & & \\
\hline 4 & $\underline{\underline{\mathrm{A}} \underline{\underline{z}, 0}}$ & $\underline{\underline{\hat{A}}}_{z, \mathrm{x}}$ & $\underline{\underline{\hat{A}_{z, y}}}$ & $\mathrm{~A}_{z, \mathrm{z}}$ & & & $\mathrm{P}_{\mathrm{z}}$ negligible for protons \\
\hline 5 & $\mathrm{~A}_{\mathrm{o}, \mathrm{xx}}$ & $\mathrm{A}_{\mathrm{o}, \mathrm{yy}}$ & $\mathrm{A}_{\mathrm{o}, \mathrm{zz}}$ & $\underline{\underline{A_{0, x y}}}$ & $\underline{\underline{\hat{A}_{0, y z}}}$ & $\underline{\underline{\hat{A}}}_{o, x z}$ & protonspinflip \\
\hline 6 & $\underline{\underline{\hat{A}}}_{\underline{x, x x}}$ & $\underline{\underline{\hat{A}}}_{\underline{\underline{x} y \underline{y}}}$ & $\underline{\underline{\hat{A}}}_{\underline{\underline{x z}}}$ & $\underline{\underline{\hat{A}}}_{x, x y}$ & $\mathrm{~A}_{\mathrm{x}, \mathrm{yz}}$ & $\underline{\underline{A_{x, x z}}}$ & $\mathrm{P}_{\mathrm{x}}$ negligible for protons \\
\hline 7 & $\underline{\underline{\hat{A}}}_{y, x x}$ & $\underline{\underline{\hat{A}}}_{y, y y}$ & $\underline{\underline{\hat{A}}}_{y, z z}$ & $\underline{\underline{\hat{\hat{A}}_{y, x y}}}$ & $\underline{\underline{A_{y, y z}}}$ & $\mathrm{~A}_{\mathrm{y}, \mathrm{xz}}$ & \\
\hline 8 & 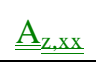 & 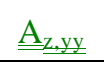 & $\underline{\underline{\underline{\mathrm{A}_{z, z Z}}}}$ & $\mathrm{~A}_{z, \mathrm{xy}}$ & $\underline{\underline{\hat{\hat{A}}}}_{z, y z}$ & $\underline{\underline{\underline{\hat{A}_{z, x z}}}}$ & $\mathrm{P}_{\mathrm{z}}$ negligible for protons \\
\hline
\end{tabular}

In Table 1, line 1 and 5 all observables cancel if $A_{y, x z}$ is calculated from a proton polarization that is flipped each time the ring is filled. Observables with a proton polarization $P_{x}$ and $\mathrm{P}_{\mathrm{z}}$ cancel too, since only the proton polarization $\mathrm{P}_{\mathrm{y}}$ is an eigenvector in the ring (the average of $\mathrm{P}_{\mathrm{x}}$ and $\mathrm{P}_{\mathrm{z}}$ should be $<10^{-8}$ in a 30 days run). This is also true in Table 1 for lines $2,4,6$, and 8 .

In the lines 3 and 7 all quantities with a hat cancel, because they are not $R_{z}$-even. $A_{y, x}$ and $A_{y, y z}$ violate parity conservation. Since $\vec{p}-\vec{d}$ scattering is an elementary process, these quantities are expected to be of the order of $10^{-7}$, even if parity is violated. Therefore, besides our quantity of interest $A_{y, x z}$, only $A_{y, y}$ "survives". Since $A_{y, y}$ in $\vec{p}-\vec{d}$ scattering is not known at $135 \mathrm{MeV}$ it has to be measured in a dedicated experiment.

The effect of $A_{y, y}$ in $\vec{p}-\vec{d}$ scattering is expected to be small, because two conditions have to be fulfilled: i) there must be a substantial deuteron vector polarization in the target, and ii) there must be a misalignment between the COSY beam direction and the deuteron beam. Only then a $\mathrm{P}_{\mathrm{y}}$ deuteron vector polarization can be generated in the target-cell. The deuteron vector polarization can be adjusted to be close to zero in the atomic beam source. The deuteron vector polarization can be continuously monitored in the dump of the atomic beam source.

Assuming the deuteron vector polarization can be limited to 0.01 , and the deuteron source and the proton beam can be aligned to better than $0.1^{\circ}$, then a false deuteron vector polarization of $\mathrm{P}_{\mathrm{y}}<2 \cdot 10^{-5}$ results. If $\mathrm{A}_{\mathrm{y}, \mathrm{y}}$ in $\overrightarrow{\mathrm{p}}-\overrightarrow{\mathrm{d}}$ scattering is $<0.05$ (from phase shift analysis estimations) the error contribution is $<10^{-6}$.

Fig. 3 depicts in general how errors are introduced to the experiment. 


\section{Error Path}

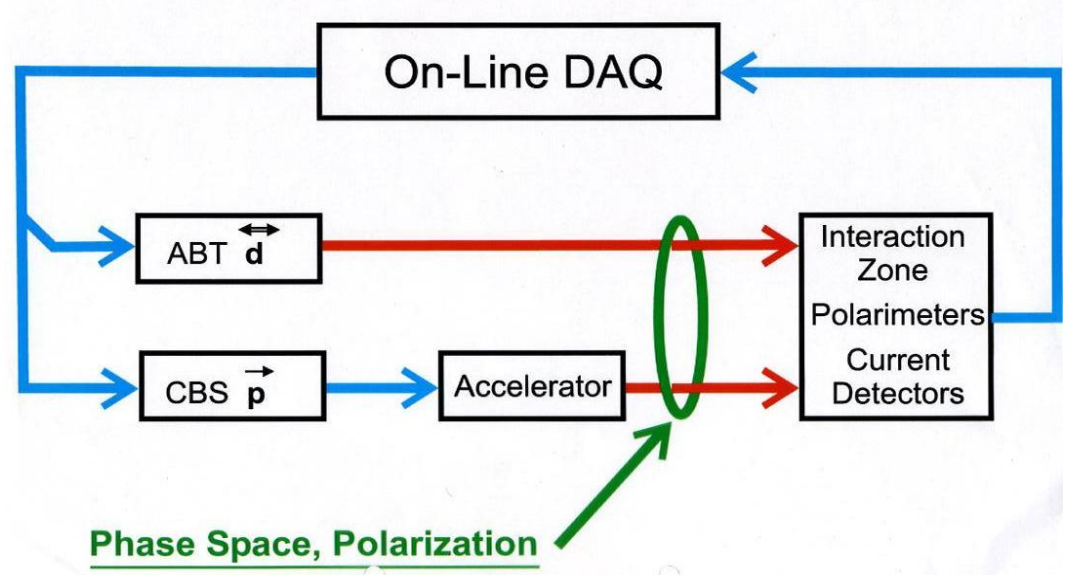

Fig. 3 Errors are introduced to the experiment by spin-flip correlated changes of beam and/or target.

The TRIC experiment can be considered as a correlation experiment in the time-domain. The interaction rate and thereof the slope of the decreasing beam current is changed only by a change of the beam properties correlated to the spin-flip of beam and/or target. Thus, the phase space including the polarizations of beam and target have to be under control. This can be achieved by redundant polarimeters and beam position monitors. Redundancy becomes important by itself. For example: The beam/target spin configuration ++ and -- as well as +- and + should give the same result. These inherent redundant cross-checks together with the unique P-even T-odd true null observable $A_{y, x z}$ allows to measure this quantity to an accuracy of $10^{-6}$ within 30 days.

\section{Acknowledgment}

The enduring, careful and painstaking work of the COSY team while developing the bunched beam for TRIC is greatly acknowledged.

\section{References}

[1] J.M. Cline, arXiv:hep-ph/0609145

[2] A. Sakharov, Pisma Zh.Eksp.Teor.Fiz. 5, 32 (1967)

[3] D. N. Spergel et.al., arXiv:astro-ph/0302209v3

[4] C.A. Baker et al., Phys. Rev. Lett. 97, 131801 (2006)

[5] W.C. Haxton, A. Höring and M.J. Musolf, Phys. Rev. D 50, 3422 (1994)

[6] P.D. Eversheim, B. Lorentz, Yu. Valdau for the PAX collaboration, COSY Proposal \#215

[7] H. E. Conzett, Phys. Rev. C 48, 423 (1993)

[8] M. Beyer, Nucl. Phys. A 560, 895 (1993)

[9] Yu. Uzikov PRC 92 (2015) 014002 
The Time Reversal Invariance Experiment at COSY (TRIC)

[10] C. Bourrely, E. Leader, J. Soffer, Phys. Rep. 59, 95 (1980)

[11] Bergoz Instrumentation, Parametric Current Transformer, Instruction Manual (1987)

[12] J. Murata et al., Hyperfine Interact. 225, 193 (2014)

[13] P. R. Huffmann et al., Phys. Rev. C 55, 2684 (1997) 